\title{
COMPLETELY CONTINUOUS INVERSES OF ORDINARY DIFFERENTIAL OPERATORS
}

\author{
ROBERT M. KAUFFMAN ${ }^{1}$
}

Many important properties, such as the absence of essential spectrum, follow when an ordinary differential operator comes from a differential expression such that the minimal operator has completely continuous inverse. This always happens in the case of a compact interval. On infinite intervals it happens much less frequently, however. To assure that the minimal operator is $1-1$, we analyze the question only on intervals of type $I=[a, \infty)$.

By an ordinary differential expression $\tau$ we mean an expression of type $\sum_{0}^{n} a_{k} D^{k}$, where $a_{k} \in C^{k}(I)$, and $a_{n}$ is nonvanishing on $I$. With $1<p<\infty$ and $1<q<\infty$, we define the minimal operator $T_{0, p, q}$ in the usual fashion. (See Goldberg's book for details.)

Our main result shows roughly that for formally selfadjoint differential expressions, $T_{0,2,2}$ has compact inverse if and only if the spectra of its selfadjoint extensions are completely dependent on the boundary conditions. A weaker result holds for $T_{0, p, p}$ with $p \neq 2$.

THEOREM 1. Let $\tau$ be a formally selfadjoint differential expression on I. Then the essential spectrum of the minimal operator $T_{0,2,2}$ is null if and only if $T_{0,2,2}^{-1}$ is completely continuous from range $T_{0,2,2}$ into $L_{2}(I)$.

Proof. Suppose $T_{0,2,2}$ has no essential spectrum. Pick a selfadjoint extension $T$ of $T_{0,2,2}$ such that $T$ is $1-1$. $T$ will have no essential spectrum. Then $T=\sum_{1}^{\infty} \lambda_{i} P_{i}$ by the spectral theorem, where each $P_{i}$ is a projection on a finite-dimensional subspace and the $\lambda_{i}$ do not cluster. Thus, $T^{-1}=\sum_{1}^{\infty}\left(1 / \lambda_{i}\right) P_{i}$ and $\left\{1 / \lambda_{i}\right\}$ has no cluster point except 0 . Clearly, $T^{-1}$ is completely continuous and therefore $\left[T_{0,2,2}\right]^{-1}$ is. The converse is clear. This completes the proof.

THEOREM 2. Let $\tau$ be a differential expression with formal adjoint $\tau^{+}$. Let $1 / p+1 / q=1$. If every solution of $\tau f=0$ is in $L p(I)$, and every solution of $\tau^{+} f=0$ is in $L q(I)$, then the minimal operator $T_{0, p, p}$ has completely continuous inverse.

Proof. $T_{0, p, p}^{-1} f(x)=\int_{a}^{x} K(x, y) f(y) d y$, where $K(x, y)=\sum_{1}^{n} f_{i}(x) \bar{g}_{i}(y)$, with $\tau f_{i}=0$ and $\tau^{+} g_{i}=0$. Complete continuity follows from this and the proof is completed.

Presented to the Society, January 24, 1969 under the title Compact inverses of ordinary differential operators; received by the editors December 20, 1968.

1 Some of this material is contained in the author's doctoral dissertation at Louisiana State University, written under the supervision of A. Zettl. 
THEOREM 3. If $\tau$ has uniformly bounded coefficients then its minimal operator $T_{0, p, p}$ does not have compact inverse.

Proof. Suppose $f$ is infinitely differentiable and is of compact support in $(a, \infty)$. Then each derivative of $f$ is in $L p[a, \infty)$. Further, $\|\tau f\| \leqq \sum_{0}^{n} M_{i}\|f i\| p$ where $T$ is of $n$th order and $M_{i}$ is a fixed positive member for each $i$. This inequality also holds for all translates of $f$. Pick any sequence $f_{j}$ of translates of $f$ which has no convergent subsequence in $L p(I)$. The set of $\tau f_{j}$ is bounded in $p$ norm and thus the inverse of $T_{0, p, p}$ is not completely continuous. This completes the proof.

CoROllaRY 1. If $\tau$ has uniformly bounded coefficients, it can not be true that every solution of $\tau f=0$ is in $L p$ and every solution of $\tau^{+} f=0$ is in $L q$, where $1 / p+1 / q=1$.

COROllaRY 2. If $\tau$ is classically selfadjoint and has uniformly bounded coefficients, every selfadjoint extension of $T_{0,2,2}$ has nonnull essential spectrum.

The following theorem will be used to show that we can put any real number into the spectrum of some extension $T_{0, p, p}$ if $\tau$ is classically selfadjoint and the minimal operator $T_{0, p, p}$ has completely continuous inverse.

Theorem 4. Suppose $\tau$ generates a minimal operator $T_{0, p, q}$. Suppose $T_{0, p, q}$ has closed range, and $\tau$ is of order $n$. Then the sum of the number of $L p$ solutions of $\tau f=0$ and the number of $L_{t}$ solutions of $\tau^{+} f=0$ is $\geqq n$, where $1 / q+1 / t=1$.

Proof. Let $r$ be the number of non $L_{t}$ solutions of $\tau^{+} f=0$. Let $\left\{f_{i}\right\}_{1}^{r}$ be chosen as follows: $f_{i}$ is of compact support in $(a, \infty)$, and $\int_{I} f_{i}(x) \bar{g}_{j}(x) d x=a_{i j}$, where $a_{i j}=0$ if $i \neq j, a_{i i} \neq 0$ for each $i$, and $\left\{g_{j}\right\}_{1}^{r}$ are the non $L_{t}$ solutions of $\tau^{+} f=0$. Further assume $\int_{I} f_{i}(x) \Psi(x) d x$ is 0 for all $L_{t}$ solutions $\psi$ of $\tau^{+} \psi=0$. Now since range $T_{0, p, q}$ is closed, we know that the minimal operator $T_{0, p, q}$ is onto the orthogonal complement of the $L q$ solution space of $\tau^{+}$, and thus there are functions $h_{i}$ in $D\left(T_{0, p, q}\right)$ with $\tau\left(h_{i}\right)=f_{i}$. Since $f_{i}$ is of compact support, $h_{i}$ is an $L p$ solution of $\tau$ on an interval of form $[c, \infty)$, where $c=$ lub support $f_{i}$. Thus $h_{i}$ can be extended to an $L p$ solution $d_{i}$ of $\tau$ on all of $I$. Suppose the $d_{i}$ are linearly dependent. Then $\sum_{1}^{r} \lambda_{i} d_{i}=0 . \quad \sum \lambda_{i} h_{i}=\sum \lambda_{i}$ $\left(h_{i}-d_{i}\right) . \int_{I}\left[\tau\left(\sum \lambda_{i} h_{i}\right)\right](x) \bar{g}_{j}(x) d x=\lambda_{j} a_{j j}$. Since the $\lambda_{j}$ can not all be 0 , we have a compact support function $\sum \lambda_{i}\left(h_{i}-d_{i}\right)=\sum \lambda_{i} h_{i}$ in $D\left(T_{0, p, q}\right)$ with $\int_{I} \tau\left(\sum \lambda_{i} h_{i}\right)(x) \bar{g}_{j}(x) d x \neq 0$. This contradicts Green's formula, since the first $n-1$ derivatives of $h_{i}$ vanish at $a$ for each $i$. 
Therefore the number of $L p$ solutions of $\tau f=0$ plus the number of $L t$ solutions of $\tau^{+} f=0$ is at least the order of $\tau$, by the above theorem, where $1 / t+1 / q=1$. This completes the proof.

THEOREM 5. If $\tau$ is formally selfadjoint, and the minimal operator as considered from $L_{p}$ to $L_{p}$ has closed range, then there are nontrivial $L_{p}$ solutions of $\tau f=0$.

Proof. Under the hypotheses we see that the number of $L_{p}$ solutions of $\tau$ and the number of $L q$ solutions of $\tau$ add up to a number which is at least $n$, the order of $\tau$. Here $1 / q+1 / p=1$. If there were not any $L p$ solutions, then all solutions would be $L q$. Thus, the inverse of the minimal operator from $L q$ to $L p$ is given by $\left(T_{0, q, p}\right)^{-1} f(x)$ $=\int_{a}^{x} h_{i}(x) \bar{h}_{j}(y) f(y) d y$ where all $h_{i}$ are in $L q$. We then see that $\left(T_{0, q, p}\right)^{-1}$ is continuous and thus range $T_{0, q, p}$ is closed.

But therefore range $T_{0, p, q}$ is closed. Thus the nonexistence of $L_{p}$ solutions contradicts Theorem 4 . This completes the proof.

Now we proceed to our characterizations of minimal operators with completely continuous inverse, assuming $\tau$ is formally selfadjoint. In the $L_{2}-L_{2}$ case, by Theorem 1 the property occurs if and only if the minimal operator has no essential spectrum. Thus, if the property holds for any real number $\lambda$, there is a 1-1 selfadjoint extension $T$ of $T_{0,2,2}-\lambda I$. Since $T_{0,2,2}$ has no essential spectrum, $\lambda \notin$ spectrum $T$. Now by Theorem 5 and Lemma 9, p. 1398, Dunford and Schwartz, each of the equal deficiency indices of $\left(T_{0,2,2}-\lambda I\right)$ is at least 1 , and thus by Theorem 10, p. 1400 of the same book, there are selfadjoint extensions which include $\lambda$ in the spectrum if the property holds. Now if the property does not hold, then $T_{0, \mathbf{2 , 2}}$ has some essential spectrum, so any selfadjoint extension also has this essential spectrum.

In the case $p \neq 2$, if the property holds there is no essential spectrum and consequently there are (see Theorem VI. 4.4, Goldberg, p. 150) 1-1 surjective extensions of $T_{0, p, p}-\lambda I$ for every $\lambda$, and $\lambda$ is not in the spectrum of any of these. By Theorem 5 there are also extensions which include any real $\lambda$ in the spectrum. It is an interesting question whether the lack of essential spectrum implies complete continuity of the inverse of the minimal operator $T_{0, p, p}$, with $p \neq 2$.

\section{REFERENCES}

1. N. Dunford and J. T. Schwartz, Linear operators. Part II, Interscience, New York, 1963.

2. S. Goldberg, Unbounded linear operators, McGraw-Hill, New York, 1966.

Western Washington State College 